\title{
Detection and compensation of anomalous conditions in a wind turbine
}

\author{
S. Hur ${ }^{\mathrm{a}, *}$, L. Recalde-Camacho ${ }^{\mathrm{a}}$, W.E. Leithead ${ }^{\mathrm{a}}$ \\ ${ }^{a}$ Wind Energy 83 Control, Department of Electronic and Electrical Engineering, University \\ of Strathclyde, Glasgow, UK
}

\begin{abstract}
Anomalies in the wind field and structural anomalies can cause unbalanced loads on the components and structure of a wind turbine. For example, large unbalanced rotor loads could arise from blades sweeping through low level jets resulting in wind shear, which is an example of anomaly. The lifespan of the blades could be increased if wind shear can be detected and appropriately compensated. The work presented in this paper proposes a novel anomaly detection and compensation scheme based on the Extended Kalman Filter. Simulation results are presented demonstrating that it can successfully be used to facilitate the early detection of various anomalous conditions, including wind shear, mass imbalance, aerodynamic imbalance and extreme gusts, and also that the wind turbine controllers can subsequently be modified to take appropriate diagnostic action to compensate for such anomalous conditions.
\end{abstract}

Keywords:

Wind turbine anomaly detection, anomaly compensation, wind turbine control, Extended Kalman Filter

1 2 3

\footnotetext{
This work was supported by the European Union's Seventh Programme for research, technological development and demonstration for the Windtrust Consortium, the grant agreement No 322449 .

* Corresponding author

Email address: hur.s.h@ieee.org (S. Hur)
}

Preprint submitted to Energy

January 25, 2017 


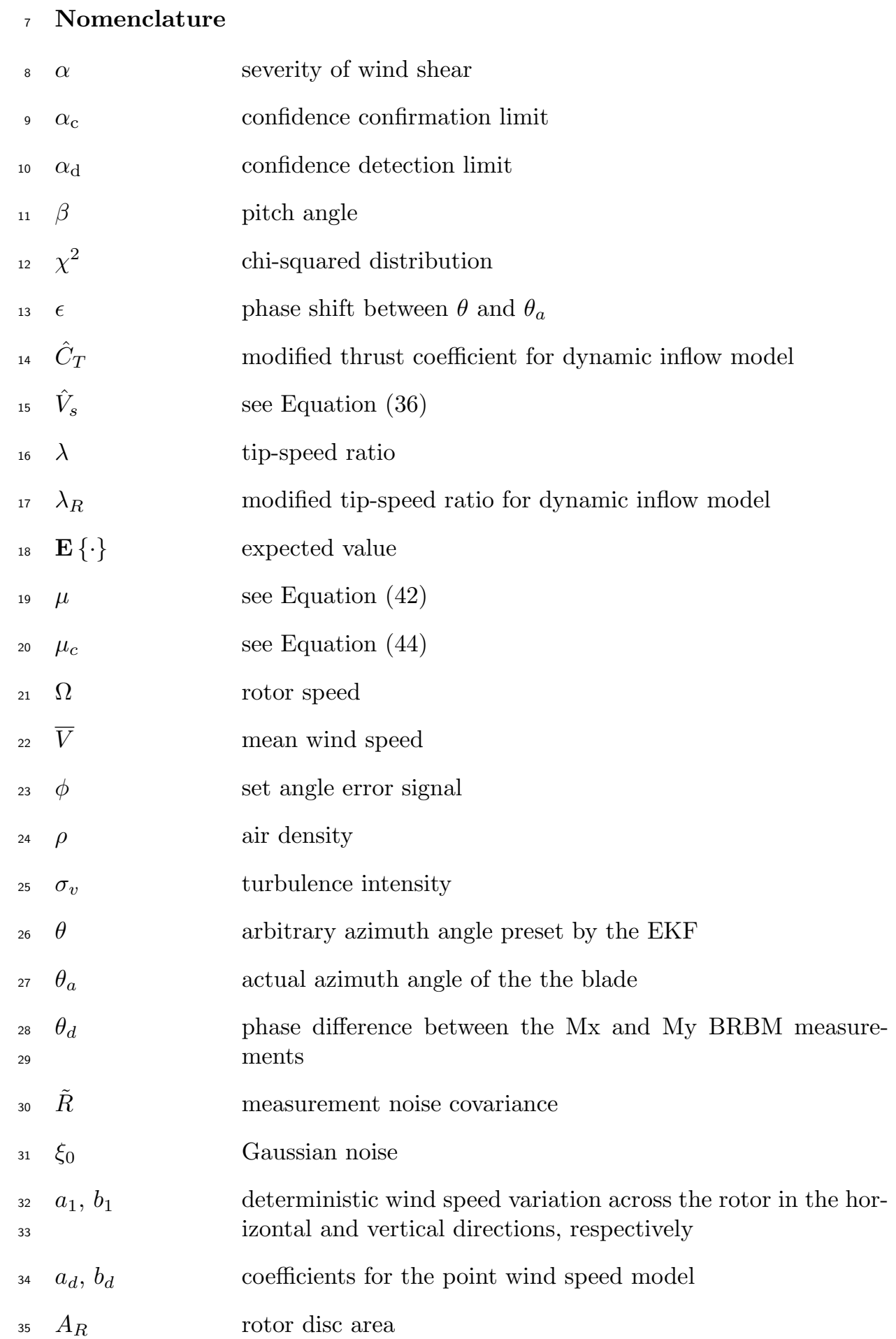




\begin{tabular}{|c|c|}
\hline${ }_{36} \quad C_{p}$ & power coefficient \\
\hline${ }_{37} C_{m x}$ & in-plane blade root bending moment coefficient \\
\hline${ }_{38} \quad C_{m y}$ & out-of-plane blade root bending moment coefficient \\
\hline $39 \quad e$ & EKF innovation error \\
\hline $40 \quad g$ & gravity \\
\hline${ }_{41} \quad g_{\mathrm{a}}$ & signature vector of the wind anomaly \\
\hline $42 \quad h$ & tower height \\
\hline $43 \quad h_{O}$ & reference height for modelling wind shear \\
\hline${ }_{44} \quad H_{\mathrm{a}}$ & signature matrix of the wind anomaly \\
\hline $45 \quad J_{f}$ & Jacobian matrix of the nonlinear function, $f\left(x_{k-1}\right)$ \\
\hline $46 \quad J_{g}$ & Jacobian matrix of the nonlinear function, $g\left(x_{k-1}\right)$ \\
\hline $47 \quad K$ & Kalman gain \\
\hline${ }_{48} \quad L_{t}$ & turbulence length of the spectrum \\
\hline${ }_{49} \quad M_{1}$ & see Equation 28 \\
\hline${ }_{50} \quad M_{2}$ & see Equation 29 \\
\hline${ }_{51} \quad M_{b}$ & blade mass \\
\hline${ }_{52} \quad M_{I / P}$ & in-plane blade root bending moment \\
\hline${ }_{53} \quad M_{O / P}$ & out-of-plane blade root bending moment \\
\hline${ }_{54} \quad N$ & number of blades \\
\hline${ }_{55} \quad N_{d f}$ & degrees of freedom \\
\hline${ }_{56} P$ & estimate error covariance \\
\hline $57 \quad Q$ & process noise covariance \\
\hline${ }_{58} \quad R$ & rotor radius \\
\hline $59 \quad S$ & EKF innovations error covariance \\
\hline${ }_{60} \quad S_{D}$ & Dryden spectrum \\
\hline${ }_{61} S_{v}$ & Von Karman spectrum \\
\hline${ }_{62} \quad T_{f}$ & aerodynamic torque \\
\hline${ }_{63} T_{\mathrm{a}}$ & anomaly starting time \\
\hline
\end{tabular}




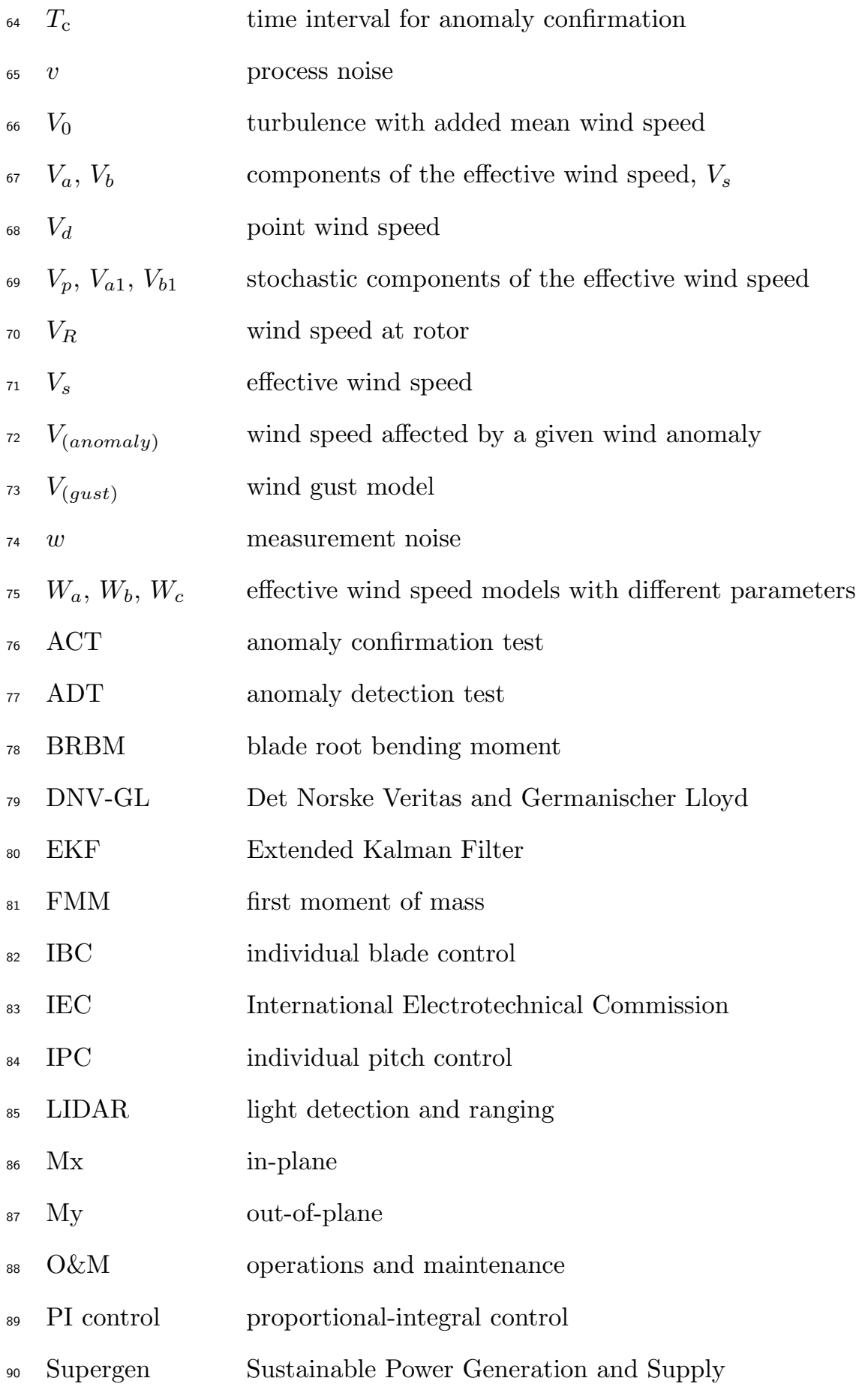


Modern wind turbine controllers usually achieve the basic objectives very well, and an anomaly detection and compensation scheme is introduced here to increase the functional capability of the (baseline) controller. Operating independently from the baseline controller, it can detect various anomalies (or anomalous conditions), including wind shear, extreme gusts, blade mass imbalance and aerodynamic imbalance. By detecting and subsequently compensating for anomalies, it would reduce the number of unnecessary shut-downs caused by the anomalies, resulting in increased energy production, and further mitigate loads on the turbine, resulting in reduced operations and maintenance (O\&M) costs or life-time extension.

The anomaly detector uses an Extended Kalman Filter (EKF) 1, 2, that is primarily based on an effective wind field model 3 and a nonlinear 3 bladed rotor (aerodynamic) model. It should be noted that the main contribution of the work is neither in the EKF nor in the models that the EKF is based on. Instead, to achieve the aforementioned novel objective, i.e. anomaly detection and compensation, a standard filtering algorithm (i.e. the EKF) and existing models are adapted.

A LIDAR (light detection and ranging) system could be utilised to achieve similar results as it allows the rotor speed and other operational control parameters to be adjusted to an approaching wind field before it reaches the turbine [4]. However, the use of a LIDAR is costly, and the model-based anomaly detection and compensation algorithm proposed here can achieve the same objective at significantly reduced cost (i.e. without a LIDAR). Hence, the main contribution of this work, in more detail, is that an anomaly detection and compensation algorithm is proposed here to increase the functional capability of the existing baseline controller at reduced cost; that is, without the need for an expensive LIDAR.

Although work on anomaly detection using a LIDAR is becoming more popular in the literature [4, 5, 6, 7, work on this topic without the use of a LIDAR is still limited [8, 9, 10. Moreover, while the work presented in 8, 9, 10, is dedicated to gust detection, the work presented here is more comprehensive dealing with various types of anomalies. It is important to note that this paper deals with "anomalies", which should be separated from "faults". Anomalies include anomalous wind conditions, such as wind shear and gusts, and structural anomalies, such as mass imbalance and aerodynamic imbalance, while faults include actuator (e.g. pitch actuator) and sensor failures. For research on fault detection, readers are referred to [11, 12, 13.

An EKF requires that the wind field model be represented in the form of a lumped parameter ordinary differential equation model. A wind field model, which meets this requirement, has been developed in 3. It outputs "effective wind speeds" for each blade and the rotor such that rotor thrust, torque and in-plane $(\mathrm{Mx})$ and out-of-plane (My) blade root bending moments (BRBMs) are represented reasonably accurately for frequencies up to the $1 \mathrm{P}$ spectral peak, which is due to rotational sampling [14, 15]. More specifically the auto and cross-spectral density functions for the forces and torques are accurate up to and including a frequency of $1 \mathrm{P}$. 


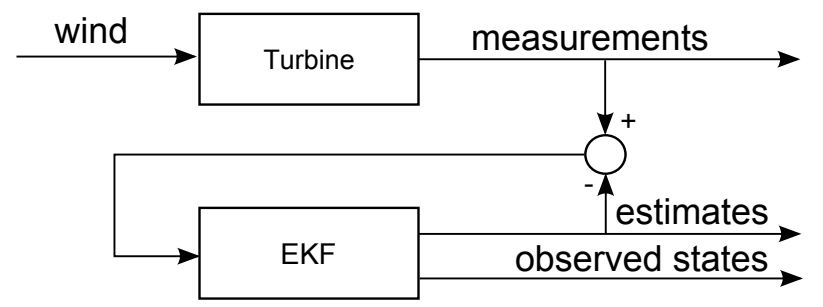

Figure 1: EKF estimates states that are not measurable or measured.

The wind field model is improved in this paper to include a model of dynamic inflow, hence to improve the accuracy of the EKF. To further improve the anomaly detection capabilities, this wind-field model could, if necessary, be extended to increase the accuracy to higher multiples of $\mathrm{P}$ at the cost of increased computational demand.

A nonlinear 3 bladed aerodynamic model, suitable for use with the wind field model, employs standard aerodynamic coefficient models for rotor torque and $\mathrm{Mx}$ and My BRBMs 3. The coefficients, which are functions of wind speed, tip-speed ratio, pitch angle, etc, are derived using Det Norske Veritas and Germanischer Lloyd's (DNV-GL) Bladed. It also takes into account the gravitational loading on the blades.

The tower dynamics could be included in the model for designing the EKF, but in return the complexity of the EKF would increase and become more computationally demanding. In any case, it does not seem to be necessary since the EKF still performs satisfactorily without the tower dynamics included in the model, as the results throughout the paper demonstrate.

The EKF is designed to track measurements of aerodynamic/hub torque and Mx and My BRBM of each blade and, in turn, to provide estimates of the states of the wind field and the gravitational loading, etc. on the blades as depicted in Figure 1. By monitoring these estimates (which are not measured or measurable in real life), various anomalies can be detected.

To enable detection of gusts, the anomaly detector is extended. Detection of gusts, unlike other anomalies, cannot be achieved by directly monitoring states estimated by the EKF. Instead, the EKF innovations error is used to monitor changes in variable correlation. Such changes are matched through best fit to a modelled anomalous scenario, e.g. extreme operating gust or extreme coherent gust. A set of modelled anomalies are derived from equations provided by International Electrotechnical Commission (IEC) 61400-1 (under the section, Extreme Conditions) [16.

Diagnostic features are also added to the anomaly detection scheme to isolate individual anomalies, estimate their magnitudes, and compensate such anomalies, for example using Individual Pitch Control (IPC) [17, 18, Individual Blade Control (IBC) [19] or open-loop control [20, 21]. This paper focuses more on the detection part and less on the compensation part, and therefore only wind shear 
and gust compensation using IPC and open-loop control, respectively, following the detection of wind shear and gust, is reported.

The EKF based anomaly detector and the models required by it are described in Section 2. Simulation results on anomaly detection are presented in Section 3 The extension of the anomaly detector for gust detection is reported in Section 4 , and anomaly compensation is described in Section 5 . Conclusions are drawn and future work discussed in Section 6 ,

The illustrative turbine in this paper is the Supergen (Sustainable Power Generation and Supply) Wind 2 MW exemplar wind turbine, which is a 3 bladed, horizontal-axis turbine, designed for variable-speed and pitch-regulated operation.

Anomalies that are detected in this paper are blade mass imbalance, wind shear, aerodynamic imbalance and extreme (coherent and operating) gust. Mass imbalance may occur due to blade icing in cold conditions and is detected in this paper by estimating each blade mass, $M_{b}$. Wind shear, variation in wind speed across the rotor disc in the vertical direction, can be caused by various factors including low level jets and weather front. It is detected in this paper by estimating deterministic wind speed variation across the rotor in the vertical direction, $b_{1}$. Aerodynamic imbalance, which can be caused by error in blade set angle, is detected in this paper by estimating set angle error signal, $\phi$. Extreme gust can be caused by various factors including turbulence due to friction, wind shear, and solar heating of the ground. It is detected in this paper by utilising the EKF innovation error, $e$. Sections 3 and 4 demonstrate the state estimation and the anomaly detection in detail. When modelling these anomalies in Bladed, we use appropriate values to ensure that the anomalies are practical and realistic by consulting industrial experts.

\section{Anomaly Detection Scheme}

To facilitate early detection of anomalous operating conditions, an anomaly detector is developed, which allows the wind turbine controller or operator to take appropriate and timely action. It is based on an EKF, which requires accurate models. An effective wind field model and a nonlinear 3 bladed aerodynamic model are utilised for designing the EKF based anomaly detector in order to detect various anomalous conditions. These models, their validation and the design of the EKF based on these models are described in this section.

As mentioned in Section 1 1 the main contribution of the work is neither in the EKF nor in the models the EKF is based on. Instead, to achieve the novel objective, i.e. anomaly detection and compensation without the use of a LIDAR, a standard filtering algorithm (i.e. EKF) and existing models, both of which are reported in this section, are adapted.

\subsection{Wind Field Model}

The wind field model outputs azimuthally and time varying effective wind speeds, $V_{s}$, for each blade and rotor so that the rotor thrust, torque and $\mathrm{Mx}$ 


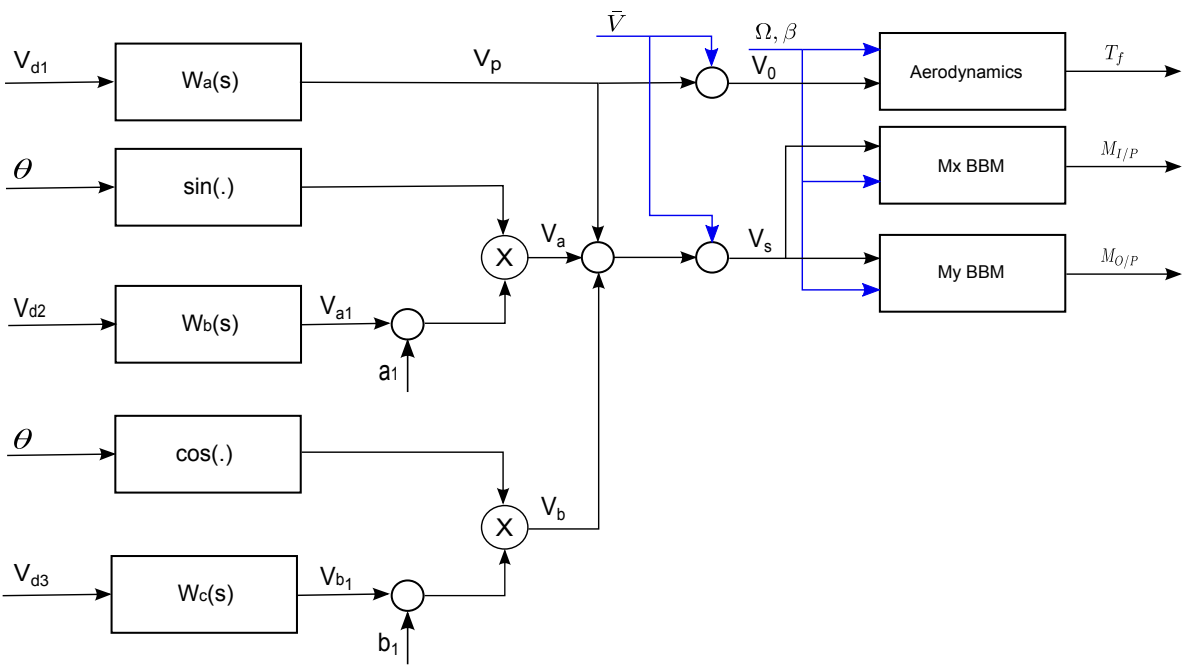

Figure 2: Wind field model and 1 of 3 blades model including frequencies up to the $1 \mathrm{P}$ spectral peak.

and My BRBMs are represented reasonably accurately at frequencies up to and including the spectral peak that corresponds to $1 \mathrm{P}$. Effective wind speed, $V_{s}$, is composed of 3 components, $V_{p}, V_{a}$ and $V_{b}$, as shown in Figure 2 More specifically, the auto and cross-spectral density functions for the forces and torques are reasonably accurate at frequencies up to and including $1 \mathrm{P}$.

The wind field on each blade consists of stochastic and deterministic components. Wind speed is in general measured on the nacelle by an anemometer, making unrealistic a direct correlation between the effects of the measured wind speeds and loads on the blades.

As the wind speed varies across the rotor, a blade element will experience different wind speeds as it rotates. The difference in wind speed across the rotor is caused by deterministic components, such as wind shear, tower shadow and blade mass imbalance, and stochastic components, such as turbulence. The wind field model represents separately the effects of the deterministic and stochastic components on the blades. The model of the wind field is depicted in Figure 2 and has the following structure [3]:

$$
V_{s}(\theta, t)=\bar{V}+V_{p}(t)+\underbrace{\left(a_{1}+V_{a 1}(t)\right) \sin (\theta)}_{V_{a}}+\underbrace{\left(b_{1}+V_{b 1}(t)\right) \cos (\theta)}_{V_{b}}+\cdots
$$

The wind speed, $V_{s}\left(\theta+\frac{2 \pi(i-1)}{3}, t\right)$, induces the moments on blade $i$ (for $i=$ $1, \ldots, N)$ where $N$ is the number of blades. $\bar{V}$ denotes the mean wind speed, and $V_{p}, V_{a 1}$ and $V_{b 1}$ are coloured noise processes representing the stochastic terms. More specifically $V_{p}$ is associated with turbulence (refer to 3 for further details), and $V_{a 1}$ and $V_{b 1}$ are respectively used to generate $V_{a}$ and $V_{b}$ that 


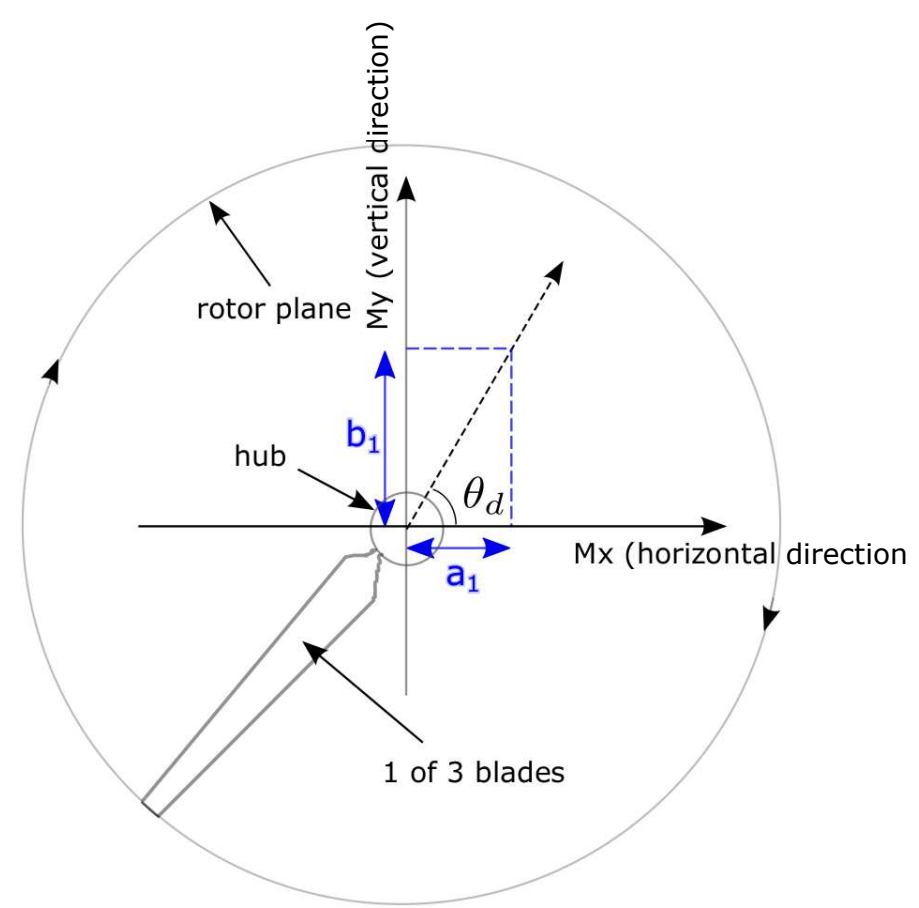

Figure 3: $a_{1}$ and $b_{1}$; variations in the horizontal and vertical axes.

eventually add the 1P spectral peak to Mx and My BRBMs.

As depicted in Figure 2, $a_{1}$ and $b_{1}$ denote deterministic variations in wind speed across the rotor in the horizontal and vertical directions, respectively. The transfer functions, $W_{a}(s), W_{b}(s)$ and $W_{c}(s)$, are part of the wind field model that have point wind speed inputs, $V_{d i}$ (for $i=1,2,3$ ), and outputs effective wind speed components [22, i.e. $V_{p}, V_{a 1}$ and $V_{b 1}$. The modules (or sub-models) on the right-hand side of the figure are explained in the following sub-section.

The deterministic components on My BRBM is dominated by wind shear (i.e. vertical variation in wind speed), while that on Mx BRBM is dominated by gravity. Gravity is at its maximum value at the blade horizontal position while wind shear causes the wind speed to be at its maximum value at the blade vertical position. When the situation is free of nacelle tilting, yaw misalignment, etc., the phase difference between the Mx and My BRBM measurements, i.e. $\theta_{d}$ in Figure 3 , should be close to $90^{\circ}$. However, the wind turbine always has such aspects, and $\theta_{d}$ can be calculated as

$$
\tan \theta_{d}=\frac{b_{1}}{a_{1}}
$$

As discussed later in Section 3, $\theta_{d}$ is an important parameter that can be monitored to detect various anomalies, including wind shear and wind veer, which 
236 are vertical and horizontal differences in wind speed, respectively.

The point wind speeds, i.e. $V_{d i}$ (for $i=1,2,3$ ), in Figure 2 can be described in the frequency domain by the Von Karman spectrum [22]:

$$
S_{v}(\omega)=0.476 \sigma_{v}^{2} \frac{\frac{L_{t}}{\bar{V}}}{\left(1+\left(\frac{\omega L_{t}}{\bar{V}}\right)^{2}\right)^{5 / 6}}
$$

where $L_{t}=6.5 h$ denotes the turbulence length of the spectrum, $h$ height and $\sigma_{v}$ the turbulence intensity. In the anomaly detection scheme, it is approximated by the Dryden spectrum:

$$
S_{D}(\omega)=\frac{1}{2 \pi} \frac{b_{d}^{2}}{\omega^{2}+a_{d}^{2}}
$$

The values of $a_{d}$ and $b_{d}$, for which the Dryden spectrum best approximates the Von Karman spectrum, are

$$
\begin{aligned}
a_{d} & =1.14 \frac{\bar{V}}{L_{t}} \\
b_{d} & =\sigma_{v} \sqrt{2 a_{d}}
\end{aligned}
$$

The corresponding point wind speed model is

$$
\begin{aligned}
V_{d} & =V_{d}(s) \xi_{0} \\
& =\frac{\bar{V} b_{d}}{s+a_{d}} \xi_{0}
\end{aligned}
$$

where $\xi_{0}$ denotes Gaussian noise.

\subsection{Nonlinear 3 Bladed Aerodynamic Model}

A 3 bladed aerodynamic model is used with the wind field model introduced in Section 2.1 to calculate the in-plane (Mx) and out-of-plane (My) BRBM and the contribution to torque for each blade. The model for one of the 3 blades contained in the complete model together with the wind field model is shown in Figure 2, where $\Omega$ is rotor speed and $\beta$ pitch angle.

Aerodynamic torque, $T_{f}$, is estimated in the module named "Aerodynamics" in Figure 2 using

$$
T_{f}=\frac{1}{2} \rho \pi V_{0}^{2} R^{3} \frac{C_{p}(\lambda, \beta)}{\lambda}
$$

where $\beta$ is pitch angle, and the tip-speed ratio, $\lambda$, is defined as

$$
\lambda=\frac{R \Omega}{V_{0}}
$$

$R$ denotes the rotor radius, $C_{p}$ the aerodynamic power coefficient and $\rho$ the air density. The parameters of the 2MW Supergen exemplar turbine are used. 
The resulting wind speed, $V_{s}$, is used by the the modules named "BBM Mx" and "BBM My", respectively, in Figure 2 for estimating the Mx and My BRBM. Similar equations to Equation (9) are utilised for these modules as follows:

$$
\begin{aligned}
M_{I / P} & =\frac{1}{2} \rho \pi V_{s}^{2} R^{3} \frac{C_{m x}(\lambda)}{3}+g M_{b} \cos \theta_{a} \\
M_{O / P} & =\frac{1}{2} \rho \pi V_{s}^{2} R^{3} \frac{C_{m y}(\lambda)}{3}
\end{aligned}
$$

Note that these equations are valid when $V_{s}$ from the wind field model described in Section 2.2 is used. $C_{m x}$ and $C_{m y}$ are respectively in-plane and out-of-plane bending root moment coefficients 3 , $g$ gravity, $M_{b}$ the first moment of mass (FMM) of each blade and $\theta_{a}$ the actual azimuth angle of the the blade. The second term in Equation (11) represents gravitational loading. Since gravity has little impact on My BRBM, given that the tilt angle is small, yaw misalignment is minimal, etc, it is excluded from Equation (12).

To model mass imbalance between the 3 blades, Equation (9) can simply be replaced with the sum of Equation (11) for blades 1, 2 and 3 as follows:

$$
T_{f}=M_{I / P, 1}+M_{I / P, 2}+M_{I / P, 3}
$$

Gravitational term in Equation (11) cancels out when summed in Equation (13) only if there is no mass imbalance.

\subsection{Validation}

The model developed in Matlab/Simulink ${ }^{\circledR}$ that combines the wind field model and the 3 bladed aerodynamic model described in Sections 2.1 and 2.2. respectively, is validated against the aero-elastic Bladed model for the same turbine, the 2MW Supergen exemplar turbine. The former model is used for developing the EKF in Section 2.4.

Simulations are run for $400 \mathrm{~s}$ with a mean wind speed of $8 \mathrm{~m} / \mathrm{s}$ and turbulence intensity of $10 \%$. The power spectra of Mx and My BRBM from the model are presented in comparison to that from the Bladed model in Figures 4 and 5. Note that hub torque is the sum of Equation (11) for blade 1, 2 and 3 (see Equation (13)), and therefore the model for hub torque does not need to be validated separately. The time-series results are not very meaningful here since the Matlab/Simulink and Bladed models experience different wind speeds in time.

Figures 4 and 5 demonstrate that $\mathrm{Mx}$ and My BRBM, respectively, from the Matlab/Simulink and Bladed models have similar spectra at low frequencies especially both displaying $1 \mathrm{P}$ peak (around $1.8 \mathrm{rad} / \mathrm{s}$ ). Since the model is developed to be reasonably accurate for frequencies up to $1 \mathrm{P}$, they are not expected to be similar at high frequencies. The red plot in Figure 4 is discussed in the following section.

The EKF is designed to ensure that any remaining discrepancy is reduced even further and also that the time response (of the EKF designed on the basis of the Matlab/Simulink model) tracks the measurements from the Bladed model closely. 


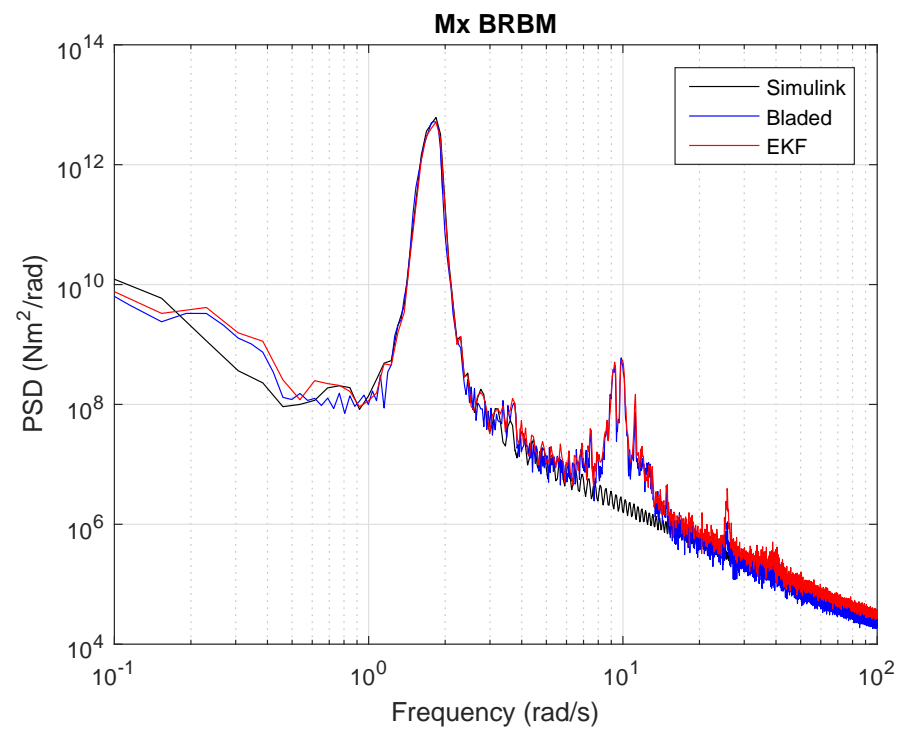

Figure 4: Power spectrum of Mx BRBM from the Matlab/Simulink model vs Bladed model vs EKF.

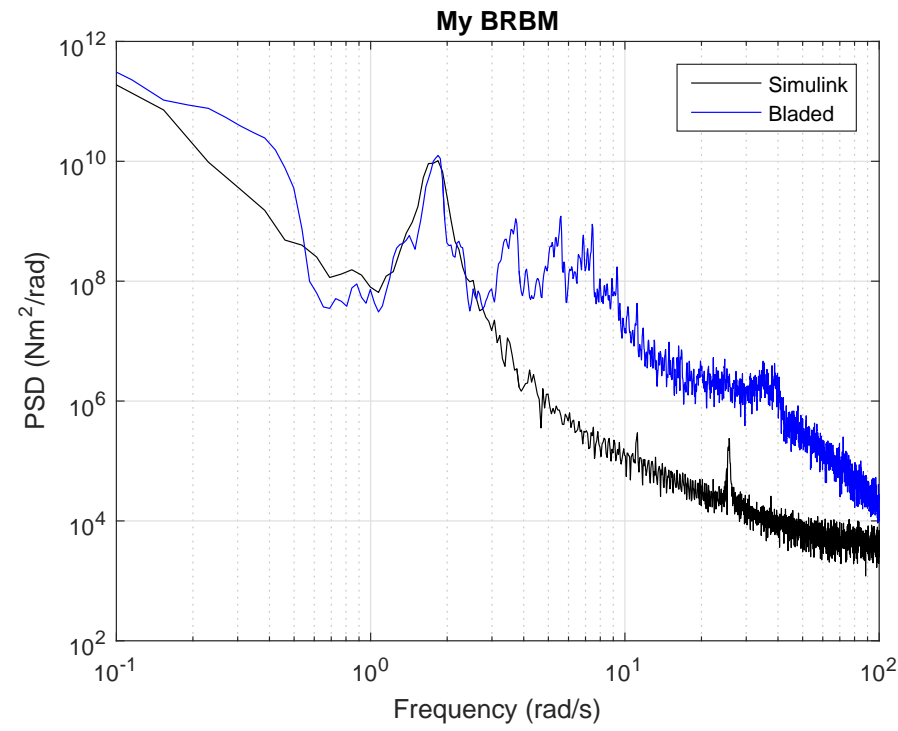

Figure 5: Power spectrum of My BRBM from the Matlab/Simulink model vs Bladed model. 


\subsection{Extended Kalman Filter}

The combined nonlinear models introduced in Sections 2.1 and 2.2 are rewritten in the following discrete form:

$$
\begin{aligned}
& x_{k}=f\left(x_{k-1}\right)+v_{k-1} \\
& y_{k}=g\left(x_{k}\right)+w_{k}
\end{aligned}
$$

where $f\left(x_{k-1}\right)$ and $g\left(x_{k}\right)$ are the nonlinear system and measurement models as described in Sections 2.1 and 2.2, respectively. For more details on the models used for $f\left(x_{k-1}\right)$ and $g\left(x_{k}\right)$, readers are referred to Appendix A. $v_{k-1}$ represents process noise, which is represented by Gaussian noise, $\xi_{0}$, from Equation (7), and $w_{k}$ denotes measurement noise. The measurement noise covariance, $R_{k}$, and the process noise covariance, $Q$, are given by

$$
\begin{array}{r}
E\left[v_{k-1} v_{k-1}^{T}\right]=Q \\
E\left[w_{k} w_{k}^{T}\right]=\tilde{R}_{k}^{T}
\end{array}
$$

$\tilde{R}_{k}$ is updated online through the use of an online covariance algorithm, while $Q$ is assumed to be constant.

The model forecast step or predictor uses the following equations:

$$
\begin{aligned}
x_{k}^{-} & \approx f\left(x_{k-1}\right) \\
P_{k}^{-} & =J_{f}\left(x_{k-1}\right) P_{k-1} J_{f}\left(x_{k-1}\right)+Q_{k-1}
\end{aligned}
$$

where $J_{f}\left(x_{k-1}\right)$ denotes the Jacobian matrix of the nonlinear function, $f\left(x_{k-1}\right)$. $x_{k}^{-}$and $P_{k}^{-}$denote the a priori state estimate and a priori estimate error covariance, respectively. The data assimilation step or corrector uses the following equations:

$$
\begin{aligned}
x_{k} & \approx x_{k}^{-}+K_{k}\left(y_{k}-g\left(x_{k}\right)\right) \\
K_{k} & =P_{k}^{-} J_{g}^{T}\left(x_{k}\right)\left(J_{g}\left(x_{k}\right) P_{k}^{-} J_{g}^{T}\left(x_{k}\right)+\tilde{R}_{k}\right)^{-1} \\
P_{k} & =P_{k}^{-}-K_{k} J_{g}\left(x_{k}\right) P_{k}^{-}
\end{aligned}
$$

where $J_{g}\left(x_{k}\right)$ denotes the Jacobian matrix of the nonlinear function, $g(x)$, and $K_{k}$ is the Kalman gain.

Since the difference between two positive-definite matrices may result in a non positive-definite matrix, which could result in numerical instability, Equation 22 is modified as

$$
P_{k}=\left(I-K_{k} J_{g}\left(x_{k}\right)\right) P_{k}^{-}\left(I-K_{k} J_{g}\left(x_{k}\right)\right)^{T}+K_{k} \tilde{R}_{k} K_{k}^{T}
$$

Now, each term in the equation is positive-definite, and $P_{k}$ is positive definite because the sum of two positive-definite matrices is positive-definite.

For more details on the formulation of EKF, readers are referred to [23, 24, 25. 


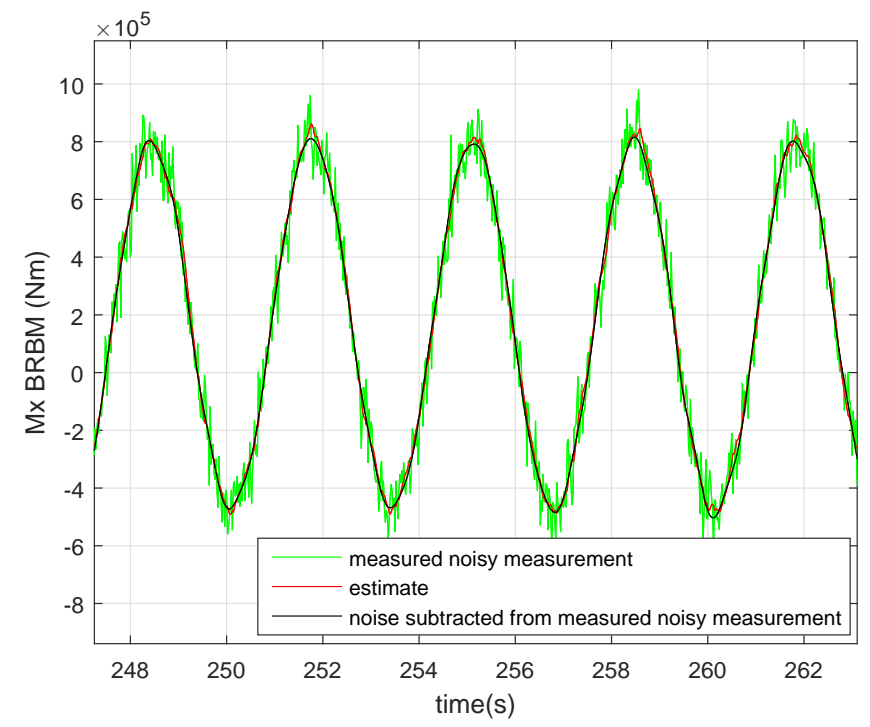

Figure 6: Noise-free tracking.

\section{Anomaly Detection}

The use of the EKF presented in Section 2 for detecting various anomalous scenarios, e.g. wind shear, mass imbalance and aerodynamic imbalance, is described with simulation results in this section. For gust detection, an extra feature needs to be incorporated into the detector, and this topic is presented in Section 4

The EKF is developed in Matlab/Simulink based on the models presented in Sections 2.1 and 2.2, but the measurements required by the EKF are obtained directly from the Bladed model that represents the same turbine, i.e. $2 \mathrm{MW}$ Supergen exemplar turbine. The Bladed model is a high fidelity aero-elastic model that is highly detailed including all the necessary blade dynamics, tower dynamics, etc. The modelling discrepancy between the two models provides a degree of model-plant mismatch to test the robustness of design. As previously mentioned, the EKF tracks measurements of hub torque and Mx and My BRBMs from the Bladed model, and provides state estimation, such as wind field components and blade mass.

To make simulations more realistic, extra noise is added to the measurements throughout this paper. An example is depicted in Figure 6, in which measurement of Mx BRBM is contaminated with noise (green), which is inputted to the EKF as opposed to the original noise-free measurement (black). Despite the added noise, the estimate by the EKF (red) is almost noise-free due to computation of the measurement noise covariance, $\tilde{R}_{k}$ in Equation (21), online by the EKF as depicted in Figure 6. The corresponding power spectra in Figure 4 (red 
and blue) also demonstrate similar characteristics in the frequency domain.

Examples of estimation of the states, the stochastic and deterministic wind speed components, $V_{p}, V_{a}$ and $V_{b}$ (see Figure 2), are depicted in Figure $7 \mathrm{a}$. These wind speed components, when aggregated, become the effective wind speed $V_{s}$ experienced by one of the blades as depicted in Figure 7b. When $V_{s}$ is used with the blade model, i.e. Equations (11) and (12), it mimics the effect of low frequency turbulence together with $1 \mathrm{P}$ rotational sampling. The EKF, at the same time, estimates other important states, such as azimuth angle, mass of each blade, etc. Monitoring of these states facilitates the detection of anomalies in various situations.

For example, the azimuth angle (i.e. angular position) and mass of each blade can be estimated and calculated as follows. The initial condition (arbitrary value predetermined by the EKF) for the azimuth angle of blade 1 is assumed to be at the 3 o'clock position by the EKF. However, the azimuth angle of blade 1 of the Bladed model (which simulates the turbine in this paper), that the EKF is monitoring, may not be at the 3 o'clock position when the EKF starts to monitor the Bladed model. The difference (i.e. phase shift) needs to be calculated and taken into account by the EKF as follows. Note, this is paramount for correctly identifying gravitational loading, wind shear, wind veer, etc as discussed below.

The gravity term in Equation (11) is rewritten as

$$
g M_{b, i} \cos \theta_{a}=g M_{b, i} \cos (\theta+(i-1) 2 \pi / 3-\epsilon)
$$

for $i=1,2,3$ ( 3 being the number of blades). $\theta_{a}$ denotes the azimuth angle of the turbine being monitored, $\theta$ the arbitrary azimuth angle preset by the EKF (i.e. at the 3 o'clock position when the EKF starts) and $\epsilon$ the phase shift such that

$$
\theta_{a}=\theta-\epsilon
$$

For blade 1, i.e. $i=1$, Equation 25 can be substituted into Equation (24) to obtain

$$
\begin{aligned}
g M_{b, 1} \cos (\theta-\epsilon) & =g M_{b, 1}(\cos \epsilon \cos \theta+\sin \epsilon \sin \theta) \\
& =M_{1} \cos \theta+M_{2} \sin \theta
\end{aligned}
$$

where

$$
\begin{aligned}
& M_{1}=g M_{b, 1} \cos \epsilon \\
& M_{2}=g M_{b, 1} \sin \epsilon
\end{aligned}
$$

$M_{1}$ and $M_{2}$ are states estimated by the EKF. These state estimates are subsequently used by the output equation, Equation (11), and also allow $M_{b}$ and $\epsilon$ to be calculated as follows:

$$
\begin{aligned}
& M_{b}=\sqrt{M_{1}^{2}+M_{2}^{2}} \\
& \epsilon=\tan ^{-1}\left(\frac{M_{2}}{M_{1}}\right)
\end{aligned}
$$




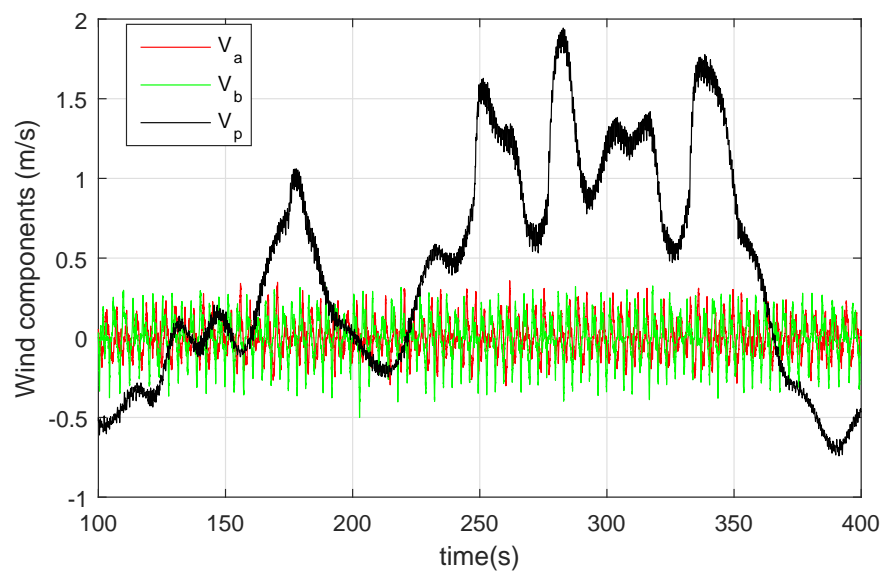

(a) Wind field components.

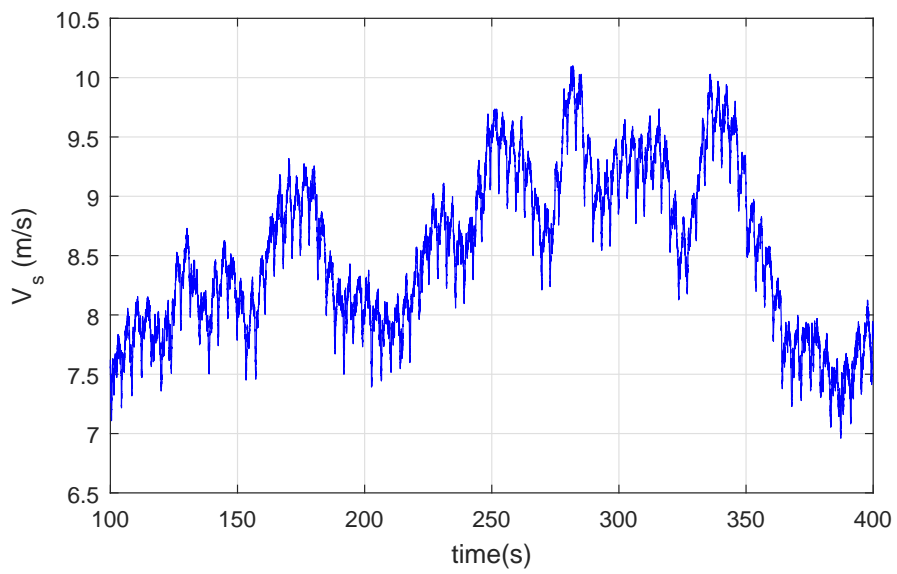

(b) Sum of the 3 wind field components.

Figure 7: Estimation of wind field components. 


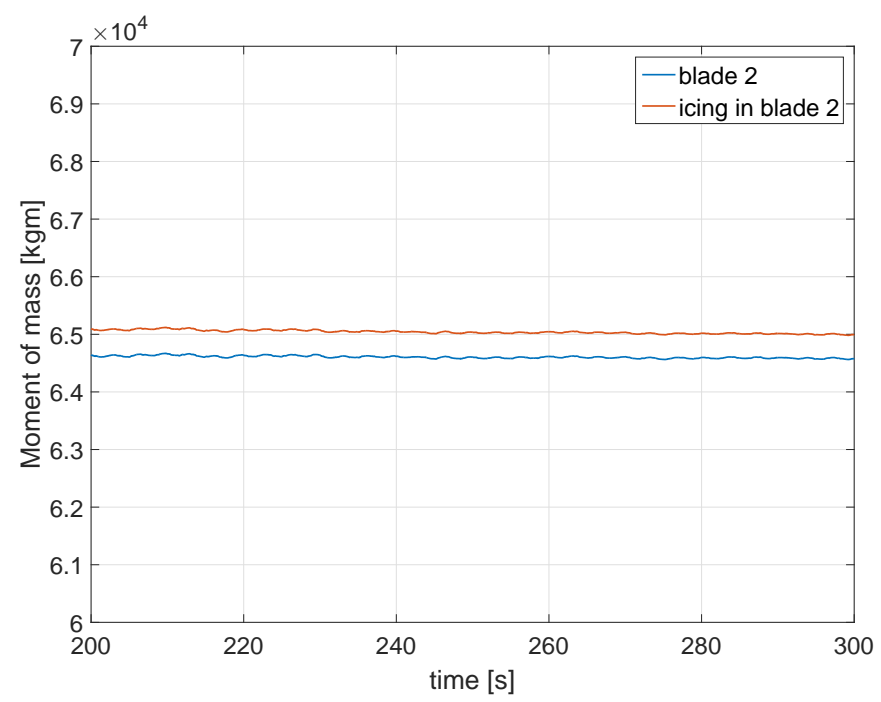

Figure 8: Mass imbalance due to blade icing.

The FMM of blade 2 estimated by the EKF is depicted in Figure 8 . The red plot is when there is $136 \mathrm{~kg}$ of ice (2.55\% of the blade mass and ice density of $700 \mathrm{~kg} / \mathrm{m} 3$ ) on blade 2 and the blue plot is when there is no ice on the blade. The estimates match the Bladed model parameters within $5 \%$. The result therefore demonstrates that the anomaly detector can be used for detecting mass imbalance, which could arise due to blade icing.

The phase shift, $\epsilon$, between the arbitrary azimuth angle and the actual azimuth angle is depicted in Figure 9. As previously mentioned, the EKF assumes that blade 1 starts at the blade horizontal position (3 o'clock). However, blade 1 of the Bladed model starts at the blade vertical position (12 o'clock). The figure demonstrates that $\epsilon$ is correctly estimated, $\epsilon=90^{\circ}$. This estimate updates the EKF, which can now be used to correctly identify gravitational loading, wind shear, wind veer, etc.

Deviations in $a_{1}, b_{1}$ and $\theta_{d}$ (in Equation (2p) from typical values can indicate anomalies in wind speed across the rotor, e.g. vertically (wind shear) and horizontally. As mentioned in Section 2.1. $\theta_{d}$ would never in reality be $90^{\circ}$ due to tilt angle, blade dynamics and so on. Note that the nacelle tilt angle is $4^{\circ}$ for the turbine considered here. $\theta_{d}$, varies with mean wind speed as depicted in Figure 10. When $\theta_{d}$ deviates from the plot in the figure, anomalies such as an increase in wind shear, wind veer or yaw misalignment can be suspected. More specifically, $b_{1}$ can be used for detecting wind shear and $a_{1}$ for detecting wind veer or yaw misalignment. An example of detecting wind shear by monitoring $b_{1}$ is given below.

As discussed in the context of Figure 3 , the state $b_{1}$ represents variation in 


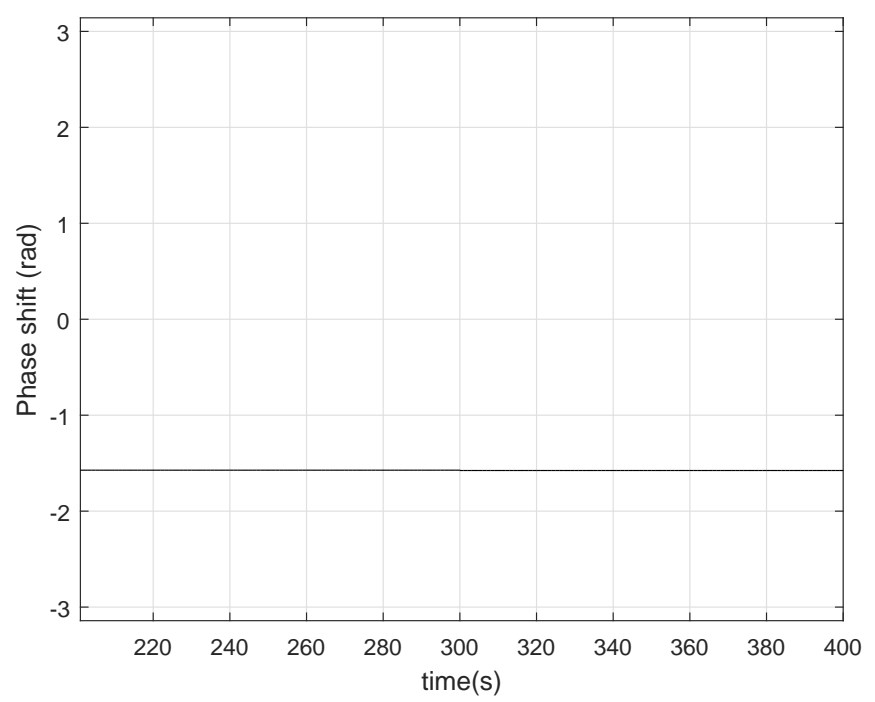

Figure 9: Phase shift.

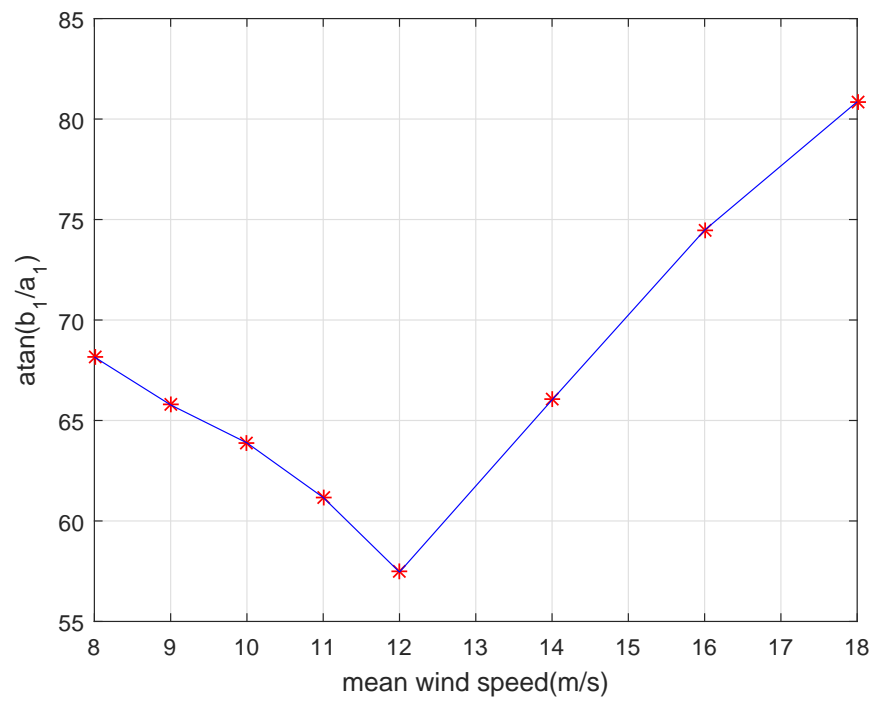

Figure 10: thet $_{d}$ at different wind speeds with tilt angle of $4 \mathrm{o}$ and wind shear with ground roughness height of $0.02 \mathrm{~m}$.

wind speed in the vertical direction, e.g. wind shear. Bladed models wind shear 


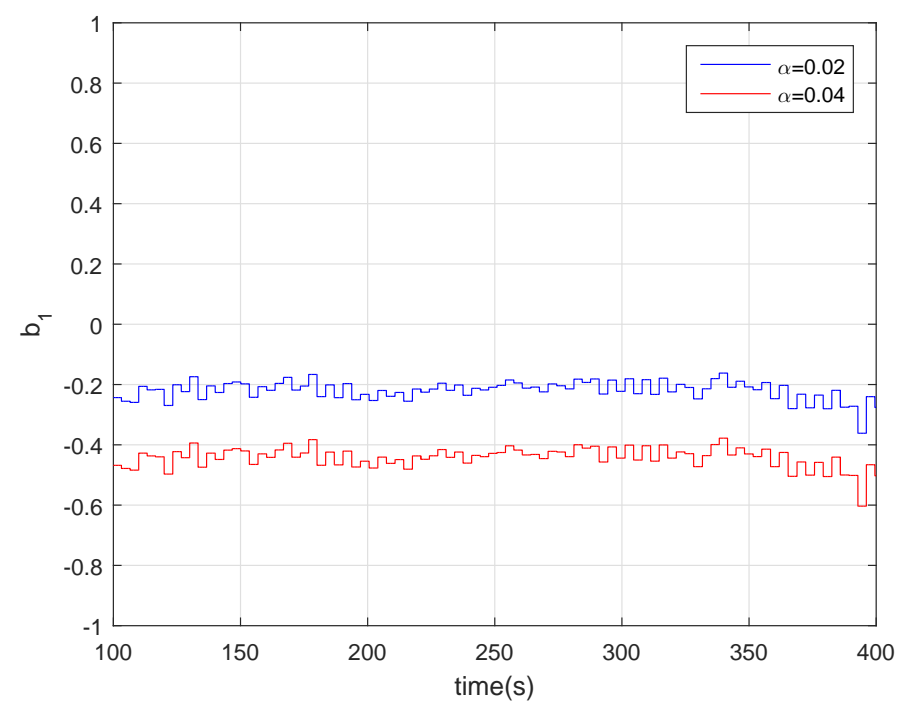

Figure 11: Wind shear.

using the following equation:

$$
V(h)=V\left(h_{o}\right)\left(\frac{h}{h_{o}}\right)^{\alpha}
$$

where $h$ denotes height above the ground and $h_{o}$ a reference height. $\alpha$ determines severity of wind shear.

Two simulations identical except for severity of wind shear are depicted in Figure 11. $\alpha$ from Equation (32) is increased by 2 times from the blue to red plots. The figure shows that monitoring $b_{1}$, estimated by the EKF, could successfully be used to detect wind shear.

The anomaly detector can also be used for detecting aerodynamic imbalance. For instance, when there is a set angle error, $\phi$, of $1^{\circ}$ in blade 2 , such that the collective pitch angle (the baseline controller acts through collective pitch angle) is slightly increased overall as depicted in Figure 12a the magnitude of the error signal (set angle error signal) between the measurement and estimate of My BRBM is increased as depicted in Figure 12b. This offset can therefore be used to detect aerodynamic imbalance, i.e. a blade set angle error in this example.

Dynamic inflow, i.e. the fractional decrease in wind speed between the free stream wind (what the wind speed would be without the turbine present) and the wind speed interacting with the turbine, continuously changes with the operating conditions. The models introduced in Section 2.1 is improved to include this effect. The following dynamic inflow model is used [26]: 


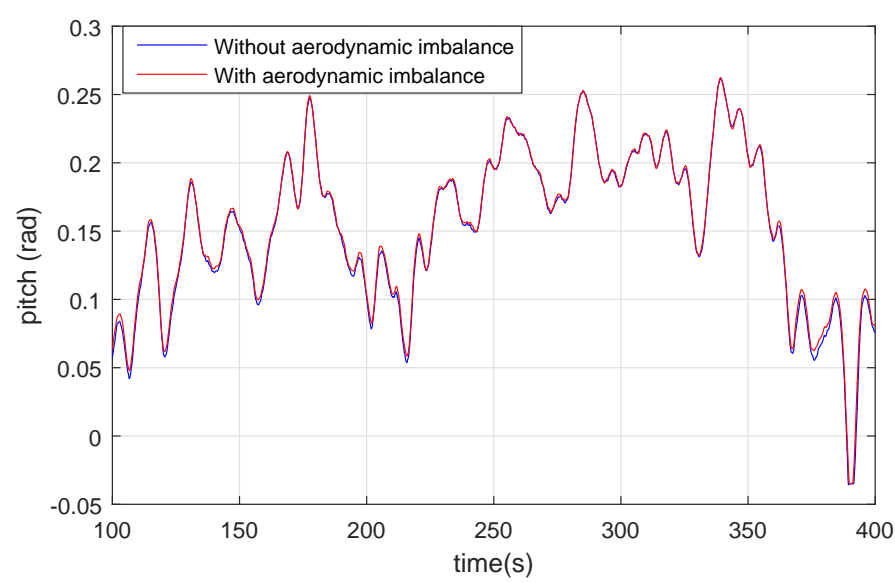

(a) Pitch with and without set angle error.

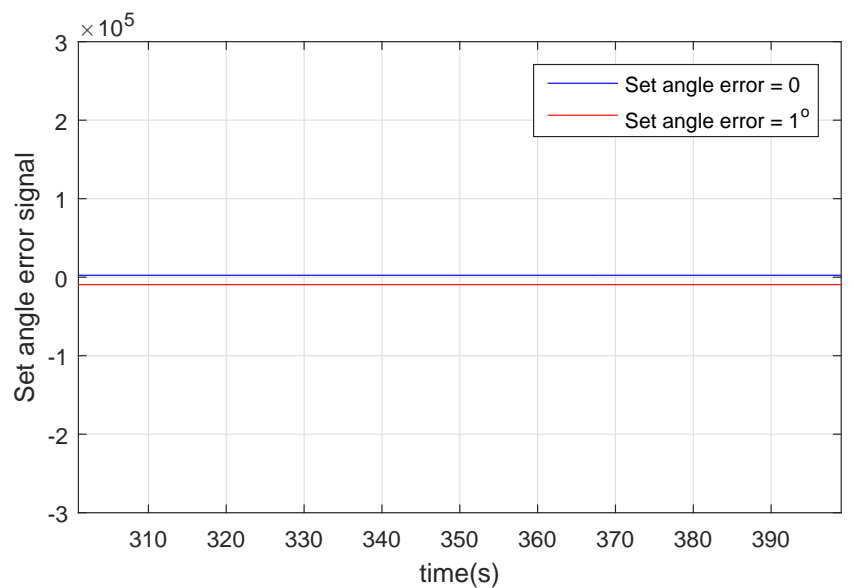

(b) Set angle error signal.

Figure 12: Aerodynamic imbalance. 


$$
\dot{V}_{R}=\frac{3}{4}\left(A_{R}\left(V-V_{R}\right) V_{R}-\frac{1}{4} A_{R} V_{R}^{2} \hat{C}_{T}\left(\lambda_{R}, \beta\right)\right) / R^{3}
$$

where $V_{R}$ denotes wind speed at rotor, $A_{R}$ rotor disc area, $V$ wind speed from Equation (1), $R$ rotor radius and $\hat{C}_{T}$ a modified $C_{T}$ table from [26. Equations (11) and (12) are modified as follows:

$$
\begin{aligned}
M_{I / P} & =\frac{1}{2} \rho \pi \hat{V}_{s}^{2} R^{3} \frac{C_{m x}(\lambda)}{3}+g M_{b} \cos \theta_{a} \\
M_{O / P} & =\frac{1}{2} \rho \pi \hat{V}_{s}^{2} R^{3} \frac{C_{m y}(\lambda)}{3}
\end{aligned}
$$

where

$$
\hat{V}_{s}=V_{R}\left(1+\frac{1}{4} \hat{C}_{T}\left(\lambda_{R}, \beta\right)\right)
$$

The incorporation of the dynamic inflow model improves the accuracy of the EKF. For instance, when the turbine switches from operating below rated to above rated, a large peak is produced on the estimate of $V_{a}$ (green) at around $390 \mathrm{~s}$ in Figure 13. This is because the effect of dynamic inflow becomes more significant when switching from operating below rated to above rated. With the dynamic inflow model properly modelled and included, the EKF now takes into account the effect of dynamic inflow, and the estimation is improved; that is, the peak is now removed (black).

\section{Extension of the Anomaly Detector for Gust Detection}

The wind field model described in Section 2.1 does not include the effects of wind gust-like events and therefore a model mismatch (between the events and the model used by the EKF) occurs in the EKF when a gust happens. Consider a model for extreme wind gusts as follows:

$$
V_{(\text {anomaly })}= \begin{cases}V_{s}(\theta, t) & t<T_{\mathrm{a}} \\ V_{s}(\theta, t) \pm V_{(\text {gust })} & t \geq T_{\mathrm{a}}\end{cases}
$$

When a gust occurs, that is, after the anomaly starting time, $T_{\mathrm{a}}$, the effective wind speed is affected by the magnitude and duration of the gust. These changes in variable correlation can be quantified by taking the expectation $(\mathbf{E}\{\cdot\})$ of the EKF innovations error, $e_{k}$, given by

$$
e_{k}=y_{k}-g\left(x_{k}\right)
$$

Expanding Equation (38) in Taylor series about $x_{k-1}$, the expectation of the innovations error is given by

$$
E\left\{e_{k} \mid y_{k}\right\}=J_{g}\left(x_{k-1}\right) J_{f}\left(x_{k-1}\right) E\left\{\tilde{x}_{k \mid k-1} \mid y_{k-1}\right\}
$$




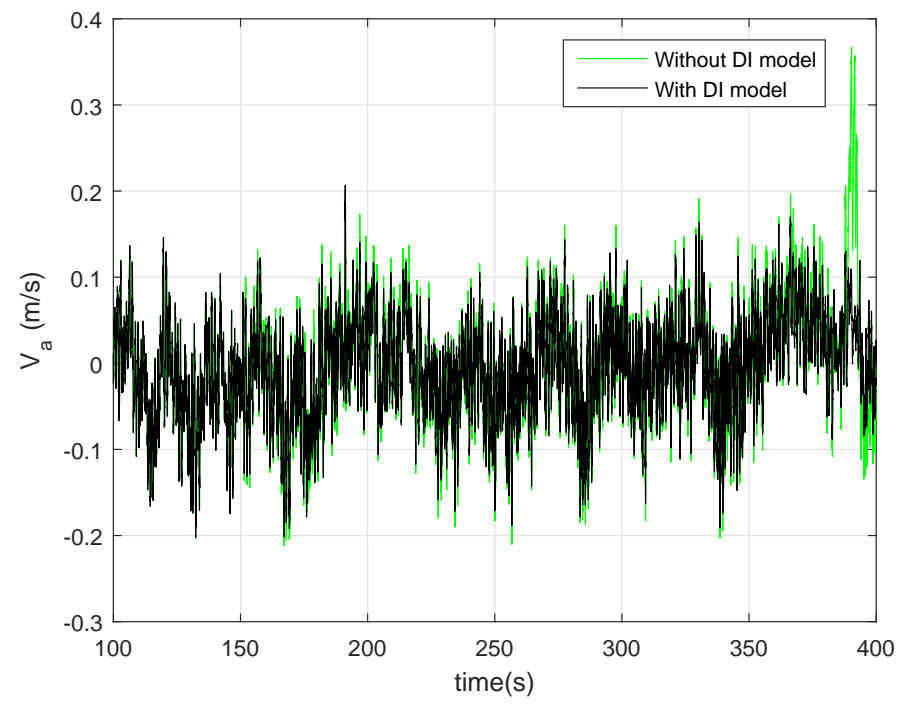

Figure 13: Dynamic inflow model.

where $\tilde{x}_{k \mid k-1}$ is the error in state estimates due to the anomaly prior to the measurement update (i.e. Equations (20), (21) and (22)) of the EKF. The calculation of state estimates posterior to the EKF measurement update can be obtained with a similar approach as follows:

$$
E\left\{\tilde{x}_{k \mid k} \mid y_{k}\right\}=J_{f}\left(x_{k-1}\right) E\left\{\tilde{x}_{k \mid k-1} \mid y_{k-1}\right\}+K_{k} E\left\{e_{k} \mid y_{k}\right\}
$$

It is therefore possible to define linear dependence of $E\left\{\tilde{x}_{k \mid k} \mid y_{k}\right\}$ on the anomaly as follow [27:

$$
E\left\{\tilde{x}_{k \mid k} \mid y_{k}\right\}=H_{\mathrm{a}}\left(k, T_{\mathrm{a}}\right) g_{\mathrm{a}}, \quad k \geq T_{\mathrm{a}}
$$

In Equation (41), the anomaly is described by signature matrix of the anomaly magnitude, $H_{\mathrm{a}}\left(k, T_{\mathrm{a}}\right)$, affecting the EKF outputs, state estimates and signature vector of its behaviour, $g_{\mathrm{a}}$. The signature matrix is time-varying allowing the magnitude of the wind gust to evolve in time. The measurement of the drift in standard deviation produced by the anomaly is determined by the Mahalanobis distance of the innovations error as follows:

$$
\mu_{k}=e_{k}^{T} S_{k}^{-1} e_{k}
$$

where $S_{k}$ is the EKF innovations error covariance given by

$$
S_{k}=J_{g}\left(x_{k}\right) P_{k} J_{g}^{T}\left(x_{k}\right)+\tilde{R}_{k}
$$

Equation 42 is used to detect unmodelled anomalies, and this process is referred to as anomaly detection test (ADT) here. The ADT follows the central 
$\chi^{2}$ distribution with $N_{d f}$ degrees of freedom and $\alpha_{\mathrm{d}}$ confidence detection limit. To avoid false alarms caused by noise, a positive ADT is followed by an anomaly confirmation test (ACT):

$$
\mu_{c, k}=\sum_{k=T_{\mathrm{a}}}^{T_{\mathrm{a}}+T_{\mathrm{c}}} e_{k}^{T} S_{k}^{-1} e_{k}
$$

The ACT follows the same distribution but has $N_{d f}\left(T_{\mathrm{c}}+1\right)$ degrees of freedom, a suitable interval time for anomaly confirmation, $T_{\mathrm{c}}$, and $\alpha_{\mathrm{c}}$ confidence confirmation limit. The following stopping rules need to be defined:

$$
\begin{aligned}
A D T_{(\text {alarm })} & =\left\{k>0, \mu_{k}>\chi_{N, \alpha_{\mathrm{d}}}^{2}\right\} \\
A C T_{(\text {alarm })} & =\left\{k>0, \mu_{k}>\chi_{N \times\left(T_{\mathrm{c}}+1\right), \alpha_{\mathrm{c}}}^{2}\right\}
\end{aligned}
$$

Practical considerations for the detection parameters are: $\alpha_{\mathrm{c}}>\alpha_{\mathrm{d}}$ and $T_{\mathrm{c}}$ longer than half the EKF convergence time. To implement a diagnostic action upon detection and confirmation of a wind gust, the signature matrix has to be estimated; that is, in order to calculate the maximum likelihood ratio in Equation (44), the signature matrix estimate is given by

$$
\begin{aligned}
H_{\mathrm{a}}\left(k, T_{\mathrm{a}}\right)= & {\left[g_{\mathrm{a}}^{T} J_{f}^{T}\left(x_{k-1}\right) J_{g}^{T}\left(x_{k-1}\right) S_{k}^{-1} J_{g}\left(x_{k-1}\right) J_{f}\left(x_{k-1}\right)\right]^{-1} \times } \\
& {\left[g_{\mathrm{a}}^{T} J_{f}^{T}\left(x_{k-1}\right) J_{g}^{T}\left(x_{k-1}\right) S_{k}^{-1}\right] g_{\mathrm{a}}^{-1} }
\end{aligned}
$$

The estimation of the signature matrix allows the detection of a wind gust at any mean wind speed. The signature vector is modelled a priori using the design standards described in [16. Goodness of fit is used to match the unknown detected anomaly to a modelled signature vector, e.g. operating wind gust or coherent wind gust. In practice, gust-like events can have any shape and magnitude. The detection begins with low goodness of fit and increases as soon as the estimated signature matrix adapts to the anomaly. The signature matrix is updated until the anomaly has passed. If the detector cannot isolate the anomaly as neither operating nor coherent gust, the anomalous data is stored and classified as unknown anomaly, thus providing the detector with an adaptability feature.

Both extreme operating gusts and extreme coherent gusts are generated in Bladed. An extreme operating gust is modelled at a mean wind speed of $14 \mathrm{~m} / \mathrm{s}$. It has the Mexican hat shape with a recurrence period of 50 year as reported in [16.

The detection of an operating gust is demonstrated in Figure 14, in which the operating gust starts at 120s. The confirmation threshold for blade $1, V_{b 1}$ (wind speed estimate for blade 1), and $\mu_{c, k}$ are included in the figure. The hub wind speed that the Bladed model experiences is also included as a reference (the Bladed model does not provide wind speed equivalent to $V_{b 1}$ ). Confidence limits for the ADT and ACT are set to 0.75 and 0.92 , respectively. Several positive alarms are triggered by noise during the ADT, and two positive alarms 


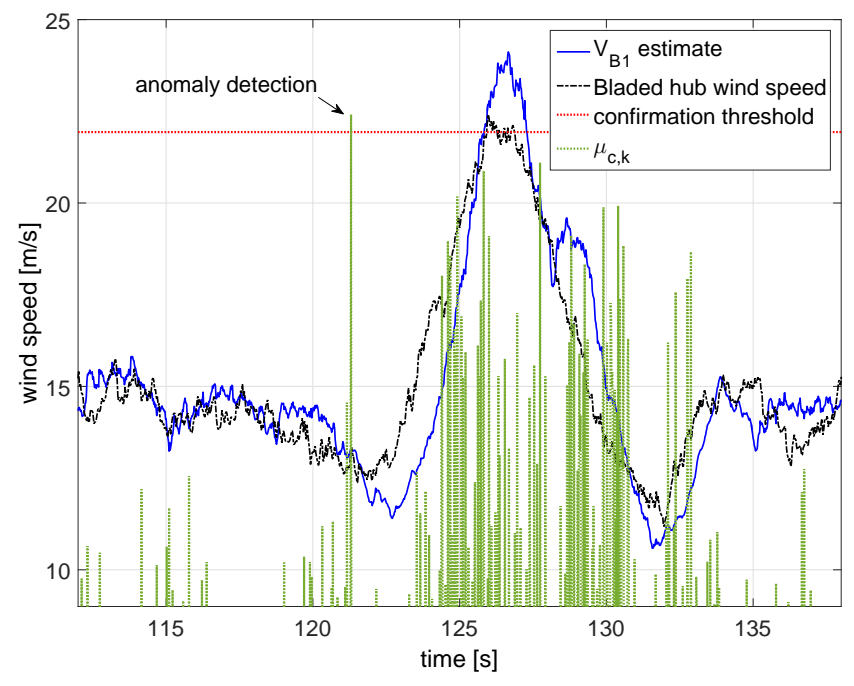

Figure 14: Extreme operating wind gust detection.

during the ACT at $64.9 \mathrm{~s}$ and $121.3 \mathrm{~s}$. The first ACT alarm does not remain to reach $T_{\mathrm{c}}$, hence the detector rules it out as false. The second alarm remains to reach $T_{\mathrm{c}}$, and thus the detector isolates it as an operating gust with a $19.8 \%$ model fit. A diagnostic action, i.e. open-loop control in this paper, can be applied at this point as described later in Section 5 . Once the signature matrix is estimated, the model fit reaches $89 \%$. This value of model fit is acceptable considering that turbulence intensity of $10 \%$ is not taken into account in the modelled wind gust.

An extreme coherent gust is modelled as a sudden cosine-shaped increase from a mean wind speed of $14 \mathrm{~m} / \mathrm{s}$ to $24 \mathrm{~m} / \mathrm{s}$, and the increase is subsequently sustained as depicted in Figure 15, in which the coherent gust starts at 73s. As with the operating gust depicted in Figure 14, the confirmation threshold for blade $1, V_{b 1}$, and $\mu_{c, k}$ are included in the figure, in addition to the hub wind speed that the Bladed model experiences as a reference. A positive ACT alarm is triggered at $74.88 \mathrm{~s}$ and negative ACT alarms at $43.48 \mathrm{~s}, 74.22 \mathrm{~s}$ and $74.48 \mathrm{~s}$. A model fit of $5.86 \%$ is initially achieved and, in turn, increases reaching $82.3 \%$. The detector can not improve the model fit further since the wind field model in the EKF is dependent on the mean wind speed, but the mean wind speed has not been updated; that is, the mean wind speed before and after the onset of the gust is different. The same diagnostic action as the one used for the operating wind gust, i.e. open-loop control, can be applied. 


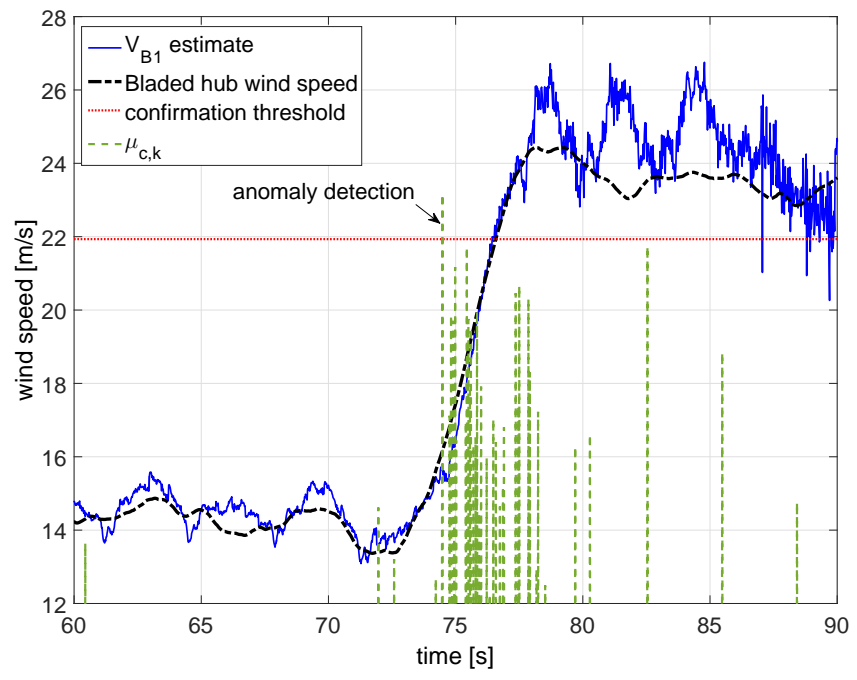

Figure 15: Extreme coherent wind gust detection.

\section{Anomaly Compensation}

Once an anomaly is detected, a remedial action (compensation) can be applied directly to the baseline controller to counteract the effects of the anomaly. The baseline controller used here is a standard commercial controller based on proportional-integral (PI) control (with modifications to incorporate fatigue reduction, anti-windup, etc.). It causes the turbine to track its design operating curve defined on the torque/speed plane 28; that is, a constant generator speed (i.e. $89 \mathrm{rad} / \mathrm{s}$ ) is maintained in the lowest wind speeds; the $\mathrm{C}_{p \max }$ curve is tracked to maximise the aerodynamic efficiency in intermediate wind speeds; another constant generator speed (i.e. $157 \mathrm{rad} / \mathrm{s}$ ) is maintained in higher wind speeds; and in above rated wind speed, the rated power of $2 \mathrm{MW}$ is maintained by active pitching. Readers are referred to [15, 29] for further details on the baseline controller.

The remedial action reported here is for wind shear and extreme operating gust. For wind shear, the baseline controller is modified to switch on IPC, which is a control technique for alleviating unbalanced rotor loads through pitching each blade separately. Additive corrections to the demanded pitch angle for each blade are determined by the controller acting on measurements of the BRBMs. This remedial action is only invoked when the anomalous behavior is detected, thus avoiding an excess of pitch activity; that is, without the anomaly detector, the IPC would need to be enabled at all times greatly increasing pitch activity and wear of the pitch actuator. For an operating gust, when the anomaly is detected, the baseline controller is modified to operate open-loop to apply maximum control actions. 


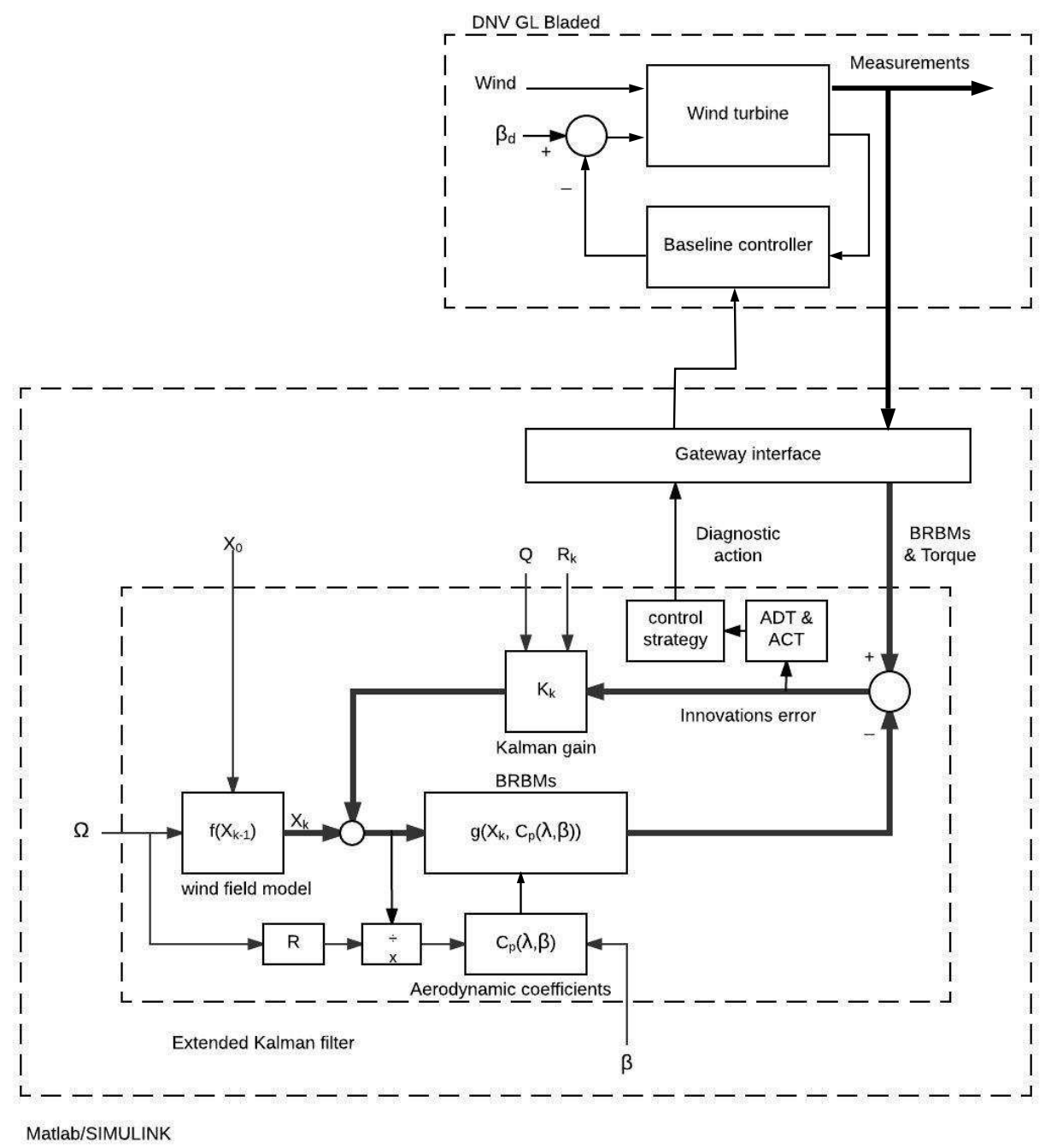

Figure 16: Anomaly detection and compensation scheme.

To apply these remedial actions on the Bladed simulation, the anomaly detector and the Bladed model are run in parallel through a commercial gateway software interface. The gateway interface allows co-simulation between Bladed and Matlab/Simulink. The simulation set-up for control compensation is presented in Figure 16. In this figure, the overall anomaly detection and compensation scheme reported throughout this paper is illustrated.

In Figure 17 from 100 to 250s, wind shear causes increased loads on the 


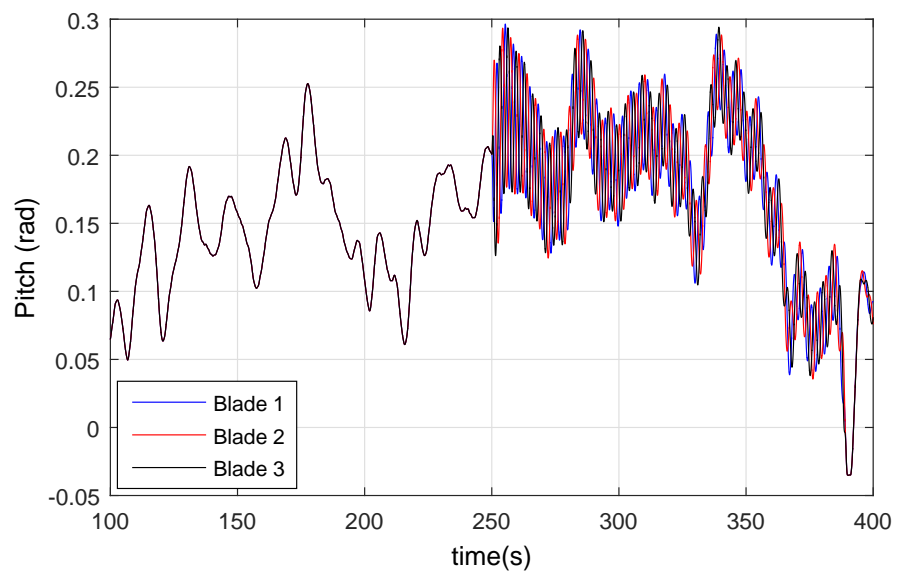

(a) IPC from 250s.

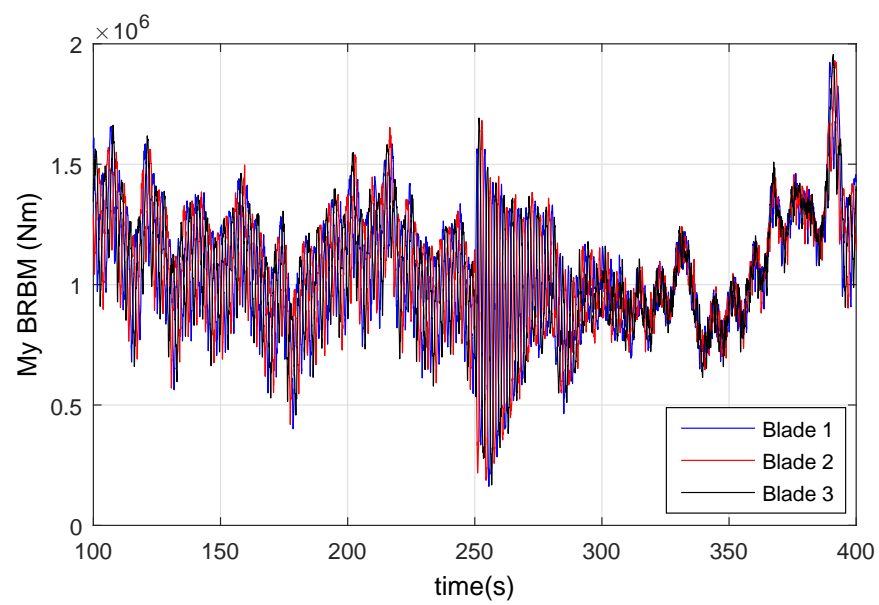

(b) My BRBM.

Figure 17: IPC compensating for wind shear. 
blades, i.e. My BRBM in Figure 17. At 250s, the anomaly detector detects an anomaly, i.e. wind shear, and thus switches on the IPC through the gateway interface as depicted in Figure 17a. Consequently, the magnitude of the oscillation on each blade is significantly decreased, resulting in reduced loads on the blades, as demonstrated in Figure $17 \mathrm{~b}$. The lifespan of the blades would thus be increased as a result of the wind shear being detected in time and appropriately compensated.

In the previous section, i.e. in Figure 14 the detection of an operating gust by the anomaly detector is described. Subsequent compensation of the gust is demonstrated in Figure 18 in this section. In Figure 14, the operating gust is detected at $121.3 \mathrm{~s}$. This allows the baseline controller to change from the normal control mode to open-loop control mode. It starts pitching at the maximum pitch rate until it is capped at $20^{\circ}$ as demonstrated (in black) in Figure $18 \mathrm{~b}$ in comparison to the situation in the normal control mode (in blue). Note that in the normal control mode, the open-loop control mode is not activated and the controller persists in following the standard control strategy described in [29]; that is, the standard commercial controller is not modified. It is shown that the baseline controller can be modified (from normal control mode to openloop control mode) in time to compensate for the anomaly. When the wind speed starts to decrease, the controller returns to the normal control mode, and as a result, rotor speed remains below the $12 \%$ threshold as shown (in black) in Figure 18c preventing the turbine from shutting down. Without the anomaly detection and compensation scheme, rotor speed exceeds the threshold as shown (in blue) in the figure. The individual turbine shut-downs not only cause reduction in the power production but also cascading shut-downs of nearby turbines, which needs to be avoided to protect the grid.

The transition from the open-loop control back to the normal control mode can significantly be improved using appropriate techniques such as the one reported in [30], but this topic is beyond the scope of this paper. 


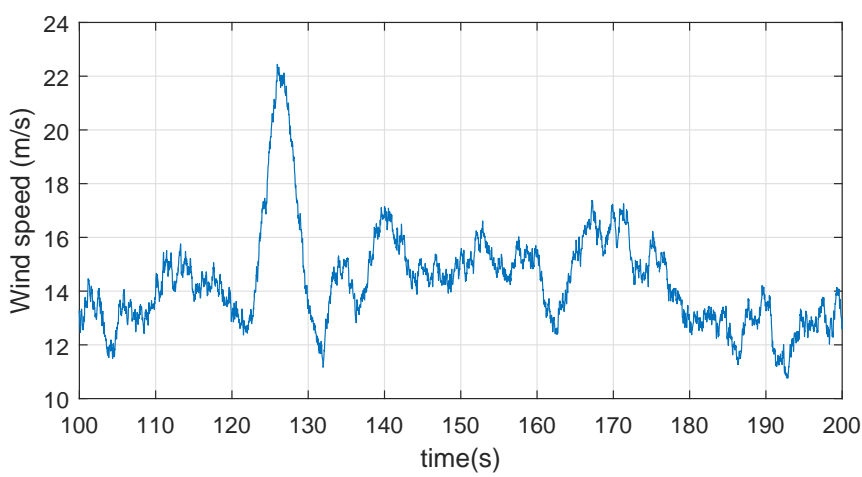

(a) Gust, the Mexican hat.

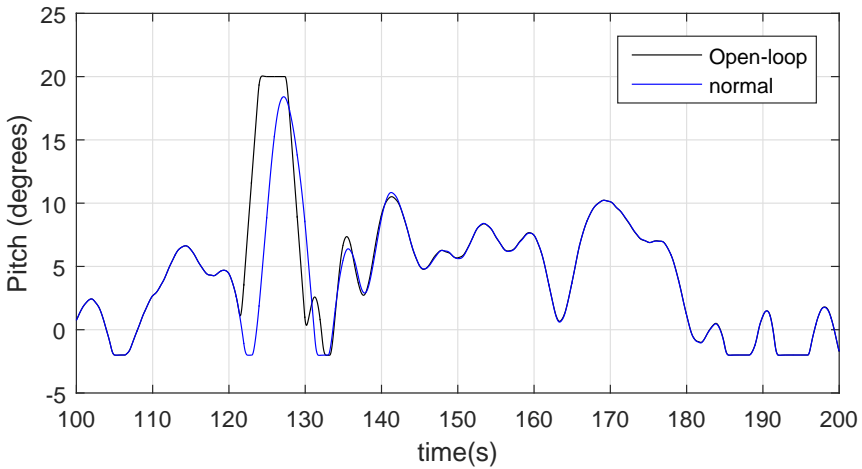

(b) Pitch.

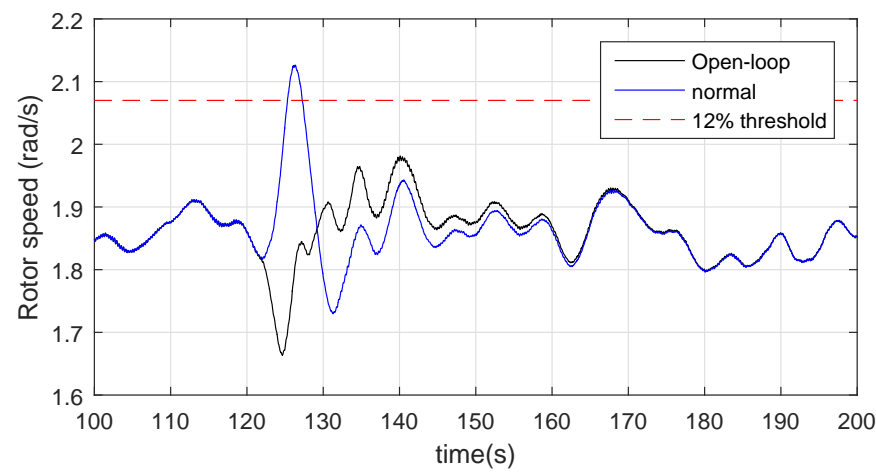

(c) Rotor speed.

Figure 18: Open-loop control compensating for gust (the Mexican hat). 


\section{Conclusion and future work}

An anomaly detection and compensation scheme for a wind turbine is reported. By detecting anomalies and taking appropriate remedial actions in time, unnecessary shut-downs can be avoided, thereby improving energy production, and structural loads can be reduced, thus improving O\&M costs.

The detection approach is to create a map of the wind field at the rotor disc using an EKF that is primarily based on a wind field model and a 3 bladed aerodynamic model. The wind field model is modified to include the effect of dynamic inflow. The EKF developed in Matlab/Simulink, using the parameters of the 2MW exemplar Supergen wind turbine, accepts measurements, i.e. aerodynamic torque and Mx and My BRBM, from the Bladed model of the same turbine. The modelling discrepancy between the two models provides a degree of model-plant mismatch to test the robustness of design. The EKF estimates states that are not measured or measurable. Simulation results demonstrate that the EKF closely tracks the measurements, coping with noise contamination, and that the state estimates can successfully be observed for detecting various anomalies, including wind shear, mass imbalance and aerodynamic imbalance.

The anomaly detector is further extended to detect extreme gusts preventing the turbine from shutting down, which would have a number of adverse consequences. The detection is made by exploiting the EKF innovations error.

Diagnostic features are added to the anomaly detector to isolate and compensate for some anomalies, i.e. wind shear and operating gust. Simulation results demonstrate that once wind shear or operating gust is detected, remedial action is successfully applied by IPC or open-loop control, respectively.

The mitigation of the impact of anomalies by means of other control strategies is being investigated. To date, the model used in the EKF is accurate up to a frequency of $1 \mathrm{P}$, but it could be extended to higher frequency to improve detection of additional anomalous scenarios, including yaw misalignment and wind veer.

\section{Acknowledgment}

The authors wish to acknowledge the support of the European Union's Seventh Programme for research, technological development and demonstration for the Windtrust Consortium, the grant agreement No 322449. The authors are also grateful to Lourdes Gala Santos for providing the equations for the wind field model and Supergen Wind for the model of the 2MW exemplar wind turbine.

\section{A. Models for the Extended Kalman Filter}

The EFK requires a discrete state space equation as described in Equations (14) and (15), and the models or equations used for the state equations, $f\left(x_{k-1}\right)$, and the output equations, $g\left(x_{k}\right)$, are described here. 
Equations used to constitute $f\left(x_{k-1}\right)$ and their derivation are summarised as follows.

1. The point wind speed model, $V_{d}(s)$, used to produce $V_{d 1}$, is combined with $W_{a}(s)$ as follows (refer to Equation (7) and Figure 2):

$$
\begin{aligned}
F_{1}(s) & =V_{d}(s) W_{a}(s) \\
V_{p} & =F_{1}(s) \xi_{0}
\end{aligned}
$$

$V_{p}$ is one of the states, $x_{k}$, to be estimated. Now the input for the model $F_{1}(s)$ is Gaussian noise, which is also the required input for the EKF as shown in Equation (14).

2. $F_{1}(s)$ is converted into the state space form and subsequently discretised, becoming $F_{1}\left(z^{-1}\right)$ (order of 4 ), to be in the suitable format for the EKF. Using this model, the EKF estimates $V_{p}$.

3. Steps 1 and 2 are repeated for $W_{b}(s)$ and $W_{c}(s)$ to give $F_{2}\left(z^{-1}\right)$ and $F_{3}\left(z^{-1}\right)$ (both order of 7 ), respectively. Using these models, the EKF estimates $V_{a 1}$ and $V_{b 1}$.

4. The terms, $\left(a_{1}+V_{a 1}\right) \sin (\theta)$ and $\left(b_{1}+V_{b 1}\right) \cos (\theta)$, in Equation (1) are used to estimate the states, $V_{a}$ and $V_{b}$ (see Figure 2). $V_{a 1}$ and $V_{b 1}$ are estimated in Step 3 above, and $\theta, a 1$ and $b 1$ are also states estimated by the EKF as reported in Section 3. These equations are used for each blade.

5. The dynamic inflow model (Equation (33)) is discretised and subsequently used by the EKF to estimate $V_{R}$.

6. Equation (36) (which is a function of $V_{R}$ from Step 4) is used to estimate $\hat{V}_{s}$.

Equations (11), (12) and (13) constitute the output equations, $g\left(x_{k}\right)$. Equations (11) and 112) are used for each blade, hence the number output equations used by the EKF is 7 .

\section{References}

[1] G. C. Goodwin, Adaptive Filtering Prediction and Control, Dover Publications, 2009.

[2] G. Besancon, Nonlinear Observers and Applications, Springe, 2007.

[3] M. L. G. Santos, Aerodynamic and wind field models for wind turbine control, Ph.D. thesis, University of Strathclyde (2016).

[4] E. A. Bossanyi, A. Kumar, O. Hugues-Salas, Wind turbine control applications of turbine-mounted LIDAR, Journal of Physics: Conference Series 555 (2014) 012011.

[5] T. Mikkelsen, Lidar-based Research and Innovation at DTU Wind Energy - a Review:, Journal of Physics: Conference Series 524 (2014) 012007. 
[6] A. Cooperman, M. Martinez, Load Monitoring for Active Control of Wind Turbines, Renewable and Sustainable Energy Reviews 41 (2014) 189-201.

[7] L. Y. Pao, K. E. Johnson, Control of Wind Turbines, IEEE Control Systems Magazine 31 (2) (2011) $44-62$.

[8] C. Carcangiu, A. Pujana-Arrese, A. Mendizabal, I. Pineda, J. Landaluze, Wind gust detection and load mitigation usingartificial neural networks assisted control, Wind Energy 17 (7) (2014) 957-970.

[9] S.Kanev, T. van Engelen, Wind Turbine Extreme Gust Control, Wind Energy 1 (13) (2010) $18-35$.

[10] J. B. E.Nederhoorn, J. Schuurmans, Increased aerodynamic performance of wind turbines through improved wind gust detection and extreme event control, in: EWEA, Barcelona, 2013, pp. 1-9.

[11] W. Liu, B. Tang, J. Han, X. Lu, Z. He, The structure healthy condition monitoring and fault diagnosis methods in wind turbines: A review, Renewable and Sustainable Energy Reviews 44 (2015) 466-472.

[12] F. Shi, R. Patton, An active fault tolerant control approach to an offshore wind turbine model, Renewable Energy 75 (2015) 788-798.

[13] H. D. M. de Azevedo, A. M. Araújo, N. Bouchonneau, A review of wind turbine bearing condition monitoring: State of the art and challenges, Renewable and Sustainable Energy Reviews.

[14] P. Brøndsted, R. Nijssen, Advances in Wind Turbine Blade Design and Materials, Elsevier, 2013.

[15] W. Leithead, B. Connor, Control of variable speed wind turbines: Design task, International Journal of Control 73 (13) (2000) 1189 - 1212.

[16] IEC, The new standard IEC 61400-1:2005 and its effect on the load of wind turbines. Part 1: Design requirements, IEC publications, Tech. rep., International Electrotechnical Commission (2005).

[17] E. Bossanyi, Individual Blade Pitch Control for load reduction, Wind Energy 6 (2) (2002) 119-128.

[18] T. van Engelen, E. van der Hooft, Individual Pitch Control, Inventory, Tech. rep., ECN Wind Energy, ECN Petten, the Netherlands (2005).

[19] H. Yi, W. E. Leithead, Alleviation of Extreme Blade Loads by Individual Blade Control during Normal Wind Turbine Operation, in: EWEA, Copenhagen, 2012, pp. 1-9.

[20] R. C. Dorf, R. H. Bishop, Modern Control Systems, 12th Edition, Prentice Hall, 2010. 
[21] K. Ogata, Modern Control Engineering, 5th Edition, Pearson, 2009.

[22] W. E. Leithead, Effective wind speed models for simple wind turbine simulations, in: Proceedings of $14^{\text {th }}$ British Wind Energy Association (BWEA) Conference, Nottingham, 1992, pp. 321-326.

[23] M. Welling, The Kalman Filter, Tech. rep., California Institute of Technology (2010).

[24] H. Musoff, P. Zarchan, Fundamentals of Kalman Filtering: A Practical Approach, 2nd Edition, American Institute of Aeronautics and Astronautics, 2005 .

[25] M. S. Grewal, A. P. Andrews, Kalman Filtering: Theory and Practice with MATLAB, 4th Edition, Wiley-IEEE Press, 2015.

[26] A. Stock, Augmented Control for Flexible Operation of Wind Turbines, Ph.D. thesis, University of Strathclyde (2015).

[27] S. C. P. J.Prakash, S. Narasimham, A Supervisory Approach to Faulttolerant Control of linear multivariable systems, Ind. Eng. Chem. Res 9 (41) (2002) $2270-81$.

[28] T. Burton, D. Sharpe, N. Jenkins, E. Bossanyi, Wind Energy Handbook, John Wiley \& Sons, Ltd, 2001.

[29] A. Chatzopoulos, Full Envelope Wind Turbine Controller Design for Power Regulation and Tower Load Reduction, Ph.D. thesis, University of Strathclyde (2011).

[30] D. Leith, W. Leithead, Directly responding to peak power excursions in pitch-regulated HAWTs, in: Proceedings of the 17th British Wind Energy Association Conference, 1995, pp. 293-298. 